\title{
Chemistry, Manufacturing and Control (CMC) Evaluations of ANDA Submission in the USA
}

\author{
Akshatha G, Anoop Narayanan V*, Sandeep D S, Narayana Charyulu R \\ Department of Pharmaceutics, Nitte Gulabi Shetty Memorial (NGSM) Institute of Pharmaceutical Sciences, NITTE (Deemed to be \\ University), Paneer, Mangalore, Karnataka, INDIA.
}

\begin{abstract}
Background: The Abbreviated New Drug Application commonly referred as ANDA is an application that contains data submitted to US FDA for the review and prospective marketing authorization of generic drugs. The conduct of the reviewing of the application is done by the Division of Filing Review (DFR), Office of Regulatory Operations (ORO) in the Office of Generic Drugs (OGD). The format of ANDA: USFDA recommends the submission of ANDA applications as per ICH Common Technical Document (CTD) format. Module 3: Module 3 of CTD contains Chemistry, Manufacturing and Control (CMC) information including all the supporting details verifying that was summarised in section 2.3 which is the quality overall summary. Evaluation of ANDA: For the evaluation of an Abbreviated New Drug Application in the US, its Office of Generic Drugs (OGD) has developed a Question-based Review $(\mathrm{ObR})$ for its CMC section which is mainly focussed on the critical quality attributes. Conclusion: The implementation of Questionbased Review programme is yielding good improvements in FDA's division of Office of Generic Drugs (OGD), i.e., by advancing the review pattern of the ANDA CMC section without compromising quality and efficacy for the indication of the generic product.
\end{abstract}

Key words: USFDA, Question-based Review, Chemistry Manufacturing and control, CTD, Module 3.

\section{INTRODUCTION}

The Abbreviated New Drug Application commonly referred as ANDA is an application that contains data submitted to US FDA for the review and prospective marketing authorization of generic drugs. The applicant will be manufacturing and marketing the generic drug once the application is approved, to provide an effective, safe drug at a lower cost that is equivalent to the brand drug (innovator's drug) name it refers. ${ }^{1}$

A drug product which is comparable to an innovator brand in its form, strength, bioavailability, route of administration and indication is termed as Generic. ${ }^{2}$ The generic drug applications are usually designated as "abbreviated" because they need not require a clinical as well as preclinical studies data to establish the safety and effectiveness of the drug product. ${ }^{1}$ It only requires bioequivalence and bioavailability study to be demonstrated which are comparable to that of innovator brand drug product. ${ }^{1}$ The "bioequivalence" study demonstrates the bioavailability of a generic drug that will be compared to that of the innovator drug product. ${ }^{1}$ FDA approves a drug in case its generic version delivers the same amount of active ingredient to the patient's bloodstream as that of the innovator drug. ${ }^{2}$

The acceptance of generic drugs by the physicians, the patients and its extension largely depend upon its adherence to strict standards laid down by the U.S. Food and Drug Administration (FDA) to gain its approval. FDA approval serves as a means of assurance to patients who receive generic medication. A survey shows that generic drugs saved 1.67 trillion dollars from the US healthcare systems from 2007 to 2016
Submission Date: 15-12-2018; Revision Date: 06-02-2019; Accepted Date: 03-05-2019.

DOI: 10.5530/ijper.53.3.74 Correspondence:

Dr. Anoop Narayanan V, Assistant Professor, Department of Pharmaceutics, NITTE Gulabi Shetty Memorial (NGSM) Institute of Pharmaceutical Sciences, NITTE (Deemed to be University), Paneer, Deralakatte, Mangalore, Karnataka- 575018, INDIA.

Phone: +919902823433

E-mail: anoopvn84@gmail. com

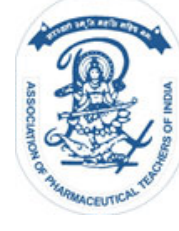

www.ijper.org 
which comprised nearly $90 \%$ of prescriptions dispensed in 2016 in the United States. ${ }^{3}$

Although generic drugs are available at a lower price as compared to that of the branded drug, a justification for their broad use must be based on the assurance of quality and substitutability to the generic drug. The generic drugs as said earlier do not require a clinical study report instead needs bioavailability and bioequivalence report. The review of the ANDA applications solely depends upon its critical pharmaceutical attributes and the bioequivalence report. $^{3}$

USFDA recommends the submission of ANDA applications as per ICH Common Technical Document (CTD) format. ${ }^{4}$

\section{Structure of Module 3 in CTD: Quality}

As shown in Figure 1 the Module 3 of CTD contains chemistry, manufacturing and control information including all the supporting details verifying that was summarised in section 2.3 which is the quality overall summary.

\section{Evaluation of the ANDA Application}

\section{Review authority}

The conduct of the reviewing of the application is done by the Division of Filing Review (DFR), Office of Regulatory Operations (ORO) in the Office of Generic Drugs (OGD). ${ }^{5}$

FDA before receiving of the ANDA applications, they are individually evaluated and the receipt of the applications means that application is complete and is permitted to the significant review. ${ }^{1}$ The complete generic application is an and A containing all the specifications required to be furnished as per the section $505(\mathrm{j})(2)(\mathrm{A})$ of the Federal Food and Cosmetics Act and devoid of deficiency as specified in 21CFR 314.101(d) and (e). ${ }^{5}$

\section{The general review process of CMC-Quality section ${ }^{5}$}

* The module 2 that contains the summaries of the data in section 2.3 comprises a Quality overall summary that summarises the information that is included in Module 3.

* Generally quality overall summary (QOS) is a Question-Based Review of which the format is provided in the PDF format and Word files.

* Module 3.2.S (Drug Substance): Review of this section of ANDA for the quality of the drug substance is based on the following documents and information.

- General information of the drug substance such as its physical and chemical properties, nomenclature structure etc.
- Check whether all required information has been submitted for all the facilities that are involved in the manufacturing and testing of the drug substance (active pharmaceutical ingredient) batches.

- Drug substance characteristics information, where the DMF reference is not acceptable.

- Complete information must be included in the subsections of 3.2.S.4 for effective review and approval.

- Complete details about on the reference standards and materials, the DMF references are not acceptable here as well.

- Stability data and container closure system information.

* Module 3.2.P (Drug Product): Regulatory review of this section of ANDA for the quality of the drug product is based on the following documents and information.

- Description of details and composition of the drug product.

- Unit compositions of each presented strength with the appropriate units.

- For all the inactive ingredients used in the proposed drug product, justifications have to be given.

- Elemental iron calculation- the FDA refuse to review (RTR) if the inactive ingredients used exceeds the limits from specified.

- Pharmaceutical development report, which includes the components of the drug product, drug product, manufacturing process development, container closure system, microbiological attributes and compatibility.

- Information on drug product manufacturer: appraise whether the information about all the facilities manufacturing and testing the commercial drug product batches.

- The batch formula for each strength of the product: evaluation is based on the comparison of the theoretical yield of the commercial batch is not more than the theoretical yield of $10 \mathrm{X}$ scale-up for that particular exhibited batch.

- Description of manufacturing process and controls: check the complete manufacturing process including their flow charts, master production batch records, master packaging records (if applicable), product sterilisation process (as applicable) and reprocessing statement.

- Assessment of those critical steps in the manufacturing process and the information on the intermediates that are submitted as a part of the requirement. Process validation or evaluation 


\subsection{Table of contents}

3.2. Body of Data

3.2.5-Drug Substance

3.2.S.1-General information

-3.2.S.1.1- Nomenclature

-3.2.5.1.2-Structure

-3.2.S.1.3-General Properties

-3.2.S.2.1- Manufacturer

3.2.5.3-Characterization: list of all potential impurities

3.2.5.4- Control of drug substances (API)

-3.2.5.4.1- Specification

-3.2.5.4.2- Analytical procedures

-3.2.5.4.3-Validation of analytical procedures

-3.2.5.4.4- Batch analysis

1. certificate of analysis (COA) and results of a

test from API manufacturer

2. certificate of analysis of Drug product

manufacture

-3.2.5.4.5-justification of specification

3.2.5.5-Materials or reference standards

3.2.5.6-Container closure system

3.2.5.7-stability

3.2.R-Regional information

3.2.R.1.S-Executed batch records for drug

substance

3.2.R.2.S-Any comparability protocols

proposed for the drug substance

3.2.R.3.S-Methods for validation package for

non-USP drugs

3.2.R.1.P-Regional information for drug

product

-3.2.1.P.1- Executed batch records for drug

product

-3.2.1.P.2-Contains information on

components but not limited to applicants

and suppliers'

- COAs drug substance lots, inactive

ingredient lot and packaging components

lots

3.2.R.2.P-Comparability protocol if applicable

3.2.R.2.P-Methods of validation package

3.2.A-Appendices
3.2.P-Drug product

3.2.P.1-Description and composition of the drug product

3.2.P.2-Pharmaceutical Development

3.2.P.3-Manufacture

-3.2.P.3.1- Drug product manufacturer

-3.2.P.3.2- Batch formula

-3.2.P.3.3-Description of manufacturing

process and process controls

-3.2.P.3.4- Controls of critical steps and

intermediates

-3.2.P.3.5-Process validation or evaluation

3.2.P.4-Control of excipients

-3.2.P.4.1- Specification

-1. Identification and characterization

-2. Certificate of analysis from the supplier

-3.2.P.4.2- Analytical procedure

-3.2.P.4.3-Validation of the analytical

procedure

-3.2.P.4.4- Specification justifications

3.2.P.5-Control of drug product

3.2.P.5.1- Specification

-3.2.P.5.2- Analytical procedures

-3.2.P.5.3- Validation of analytical

procedures

-3.2.P.5.4- Batch analysis

-3.2.P.5.5- Characterization of impurities

-3.2.P.5.6-Specification justification

3.2.P.6-Information regarding the reference

standards

3.2.P.7-Container Closure system

3.2.P.8-Stability

-3.2.P.8.1- finished dosage form: stability

summary and conclusion

-3.2.P.8.2- Post approval stability

commitment and stability protocol

-3.2.P.8.3- Stability data

Figure 1: Sections of module 3 of CTD. ${ }^{2}$

- Assessing the control of excipients by the information provided on the source of the inactive ingredients, specifications, analytical procedures, validation of analytical methods and justification of specifications.

- Assessing the control of drug product based on the information on specifications, analytical procedures, validation of analytical procedures, batch analysis, characterisation of impurities, justification of specifications.

- The container closure system used will be checked for their source of supplies and supplier address, components specification and test data.

- Stability test data: assessment for their expiration period post-approval stability.

* Module 3.2.R (regional): as per US regulatory authority the following data are to be mentioned in this regional information, the assessment is as follows:
- Assessment of the executed batch records for the comparison with the manufacturing and packaging relationship.

- Information about the components

- As per applicability comparability of the protocols

- Assessment of methods of validation if applicable.

\section{Question-Based Review (QBR) of CMC Sections}

\section{Development of $Q b R$}

For the evaluation of an Abbreviated New Drug Application in the US, its Office of Generic Drugs (OGD) has developed a Question-based Review (QbR) for its Chemistry Manufacturing and Control section which is mainly focussed on the critical quality attributes. The initiative for its implementation began in the year 2005 with a revised review template and post receiving the feedback from the stakeholders through the wide inter- 


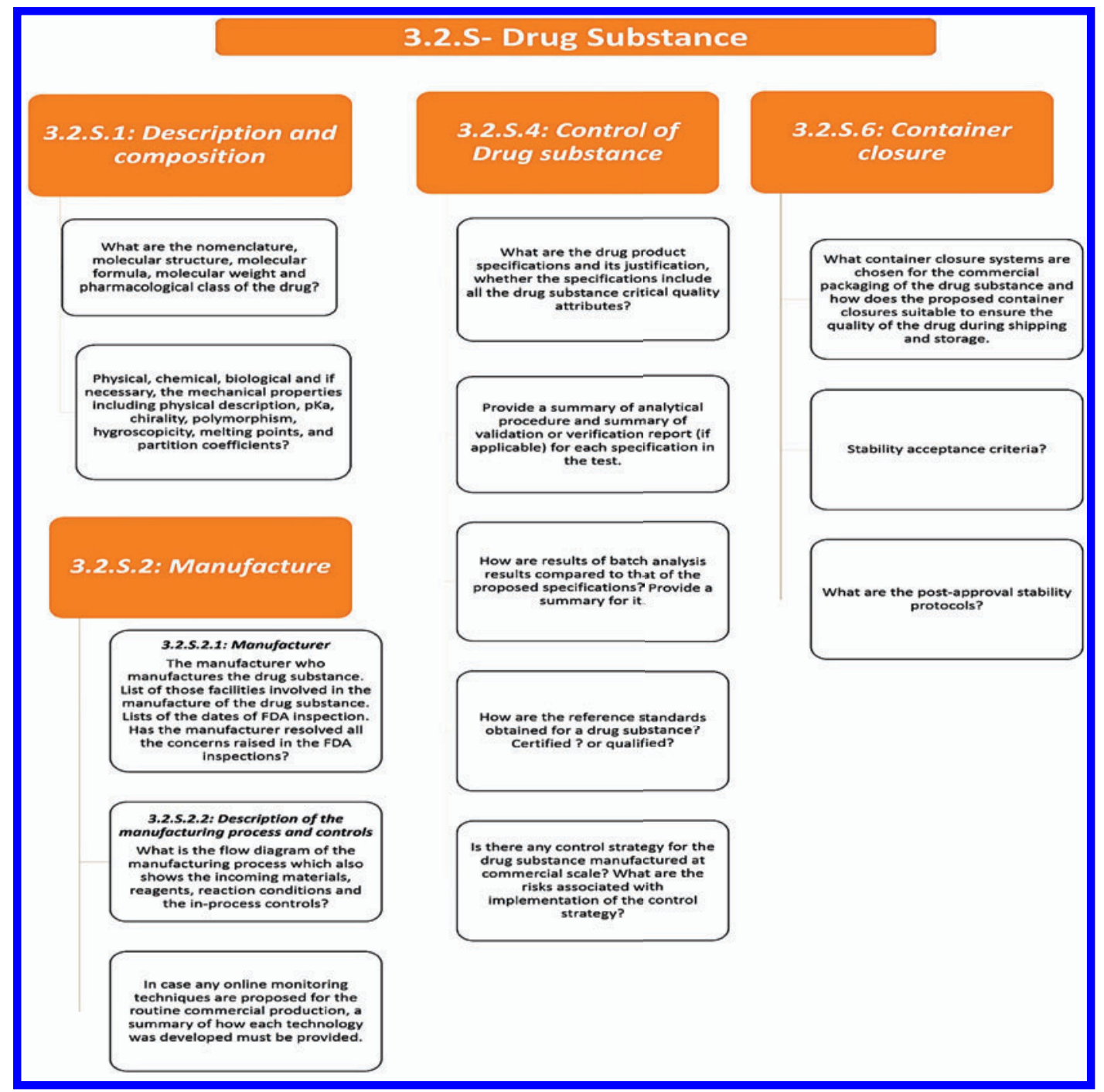

Figure 2: Questions on the section drug substance of module 3.2.

net and external discussions; it was implemented in the year $2007 .^{6}$

The Question-based review has transformed the CMC review into a modern, science and risk-based pharmaceutical quality assessment. The objectives of this question-based review system include. ${ }^{6}$

- Assuring the product quality through design and performance-based specifications

- Through risk assessment facilitate continuous improvement and reduce CMC supplements.

- Set standardized review questions to enhance the quality of reviews.

- Reduce the CMC review time.

QbR was designed and implemented that the ANDA submissions are structured according to Common Technical Document (CTD), a submission format that has been accepted by some regulatory agencies com- prising of USFDA as well. CTD format facilitates the implementation of QbR and the review of applications without any undue delays. ${ }^{2}$

\section{QbR based review for CMC submissions ${ }^{7}$}

QbR questions were framed according to the ICH M4Q Common Technical Document (CTD) format, the questions are relating to each of its section (Module 3 Figure 1). These set of questions are applicable to all the kinds of dosage forms, yet some dosage form related data may be acquired from the applicant.

The reviewers of drug substance and drug product of the Office of Pharmaceutical Sciences (OPS) will be provided with a QbR template while they evaluate the ANDA submissions. The Figure 2 and 3 show the kind of question for review of section 3.2 and $3.2 \mathrm{~S}$ for the drug product and the drug substance respectively. 


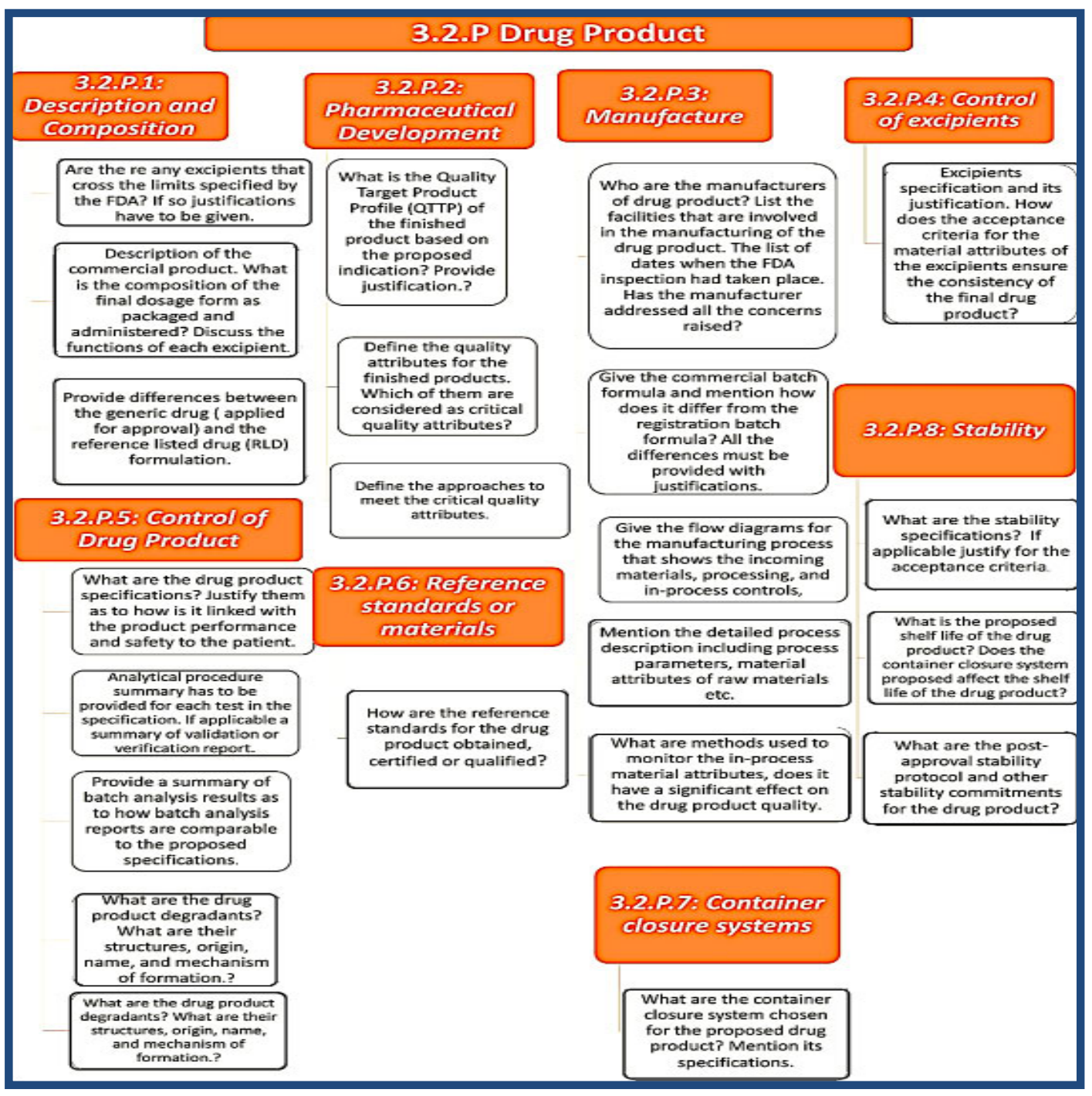

Figure 3: Questions for drug product a section under module 3.2.

Even though QbR covers the maximum of all the essential science and regulatory-based questions, the reviewers still are allowed to communicate to the applicants in case of lack of information provided or for their clarifications through any appropriate route.

\section{Procedures for the review}

The reviewers work includes:

The assessment of the submission will be carried out using the current version of the suitable template.

The applicant's response for the application must be extracted and deduced a summary of which will be provided in the review in case the submission is not a QbR format.

QbR companion documents shall be used as reference guides to complete the review process. Companion documents include some of the information that could be submitted to answer the QbR questions, while they serve only as examples which should not be considered as a template.

The information provided in the quality overall summary of Module 2 and body of the data section of Module 3 must be read correctly for the primary review and must be acquainted that the information provided in the QOS does not contradict with information provided in the body of data of Module 3 .

Some of the subsections like container closure systems and product development can be grouped as one for which a single summary may be provided.

The following are the set of questions of Questionbased Review programme that the reviewers of ANDA check through.

\section{CONCLUSION}

The implementation of Quality-based Review programme is yielding good improvements in FDA's 
division of Office of Generic Drugs (OGD), i.e., by advancing the review pattern of the ANDA CMC section without compromising quality and efficacy for the indication of the generic product. ${ }^{8}$

The series of questions in the QbR programme focuses on critical information needed to evaluate the product quality. ${ }^{8}$

Further, it is also planning to increase the staff to assess the risk-based quality approach and making every possible effort to make the generic drug reach the market. ${ }^{8}$ The systematic, modernised review of the ANDA applications enhances the quality of the review, which eventually affects the generic drug market. ${ }^{6}$ Today 9 out of 10 prescriptions in the US are generic drugs. As a result, people are able to receive medication at an affordable price. $^{9}$

\section{ACKNOWLEDGEMENT}

The authors are thankful to the principal and the management of NITTE (Deemed to be University) for their constant encouragement and support.

\section{CONFLICT OF INTEREST}

The authors declare no conflict of interest.

\section{ABBREVIATIONS}

CMC: Chemistry Manufacture and Control; ANDA: Abbreviated New Drug Application; USA: United States of America; USFDA: United States Food and Drug Administration; CTD: Common Technical Docu- ment; ICH: International Conference on Harmonisation; QbR: Question-based Review; OGD: Office of Generic Drugs; ORO: Office of Regulatory Operations; DFR: Division of Filing Review.

\section{REFERENCES}

1. Nahler G. Abbreviated new drug application. In: Dictionary of Pharmaceutical Medicine. Vienna: Springer Vienna. 2009;1.

2. Balasubramanian J, Chenchu TVY, Eknath B, Hari RS, Vinoth. Influence of (QbR) question based review to revamp CMC submission. World J Pharm Med Res. 2018;4(4):100-6.

3. Uhl K, Peters JR. How the FDA Ensures High-Quality Generic Drugs. Am Fam Physician. 2018 ;97(11):696-7.

4. Abbreviated New Drug Application (ANDA) Forms and Submission Requirements. Center for Drug Evaluation and Research. 2018. [cited 2019 Feb 6]. Available from: https://www.fda.gov/Drugs/ DevelopmentApprovalProcess/HowDrugsareDevelopedandApproved/ ApprovalApplications/AbbreviatedNewDrugApplicationANDAGenerics/ ucm120955.htm

5. Policies and procedures: Filing Review of Abbreviated New Drug Applications. USA: Manual of Policies and Procedures. 2017;1-43.

6. Question-Based Review (QbR) for Generic Drugs: An Enhanced Pharmaceutical Quality Assessment System. Center for Drug Evaluation and Research. 2018. [cited 2019 Feb 6]. Available from: https://www.fda.gov/Drugs/ DevelopmentApprovalProcess/HowDrugsareDevelopedandApproved/ ApprovalApplications/AbbreviatedNewDrugApplicationANDAGenerics/ ucm120973.htm

7. Policy and procedures: Chemistry review of question-based review (QbR) submissions. Manual of policies and procedures, MAPP 5015.10 Center of drug evaluation and research. 2014;1-12.

8. Maddela LK, Oh I. Advances in the review of generic drug applications by united states food and drug administration, a regulatory perspective in the era of GDUFA (generic drug user fee amendment 2012). Int J Drug Regul Aff. 2018;3(1):41-61.

9. FDA. Generic drugs. Information for Consumers (Drugs). Center for Drug Evaluation and Research. 2018. [cited 2018 Nov 30]. Available from: https://www.fda.gov/Drugs/ResourcesForYou/Consumers/ BuyingUsingMedicineSafely/GenericDrugs/default.htm

\section{SUMMARY}

A drug product which is comparable to an innovator brand in its form, strength, bioavailability, route of administration and indication is termed as Generic. The ANDA is an application that contains data submitted to USFDA for the review and prospective marketing authorization of generic drugs. Although generic drugs are available at a lower price as compared to that of the branded drug, a justification for their broad use must be based on the assurance of quality and substitutability to the generic drug. Module 3 of CTD contains chemistry, manufacturing and control (CMC) information including all the supporting details verifying that was summarized in section 2.3 which is the quality overall summary. A Question-based Review (QbR) is used for the evaluation of CMC section which is mainly focussed on the critical quality attributes, which serves as a science and risk-based pharmaceutical quality assessment. This method helps in enhancing the quality of review and reduces the $\mathrm{CMC}$ review time. 
PICTORIAL ABSTRACT

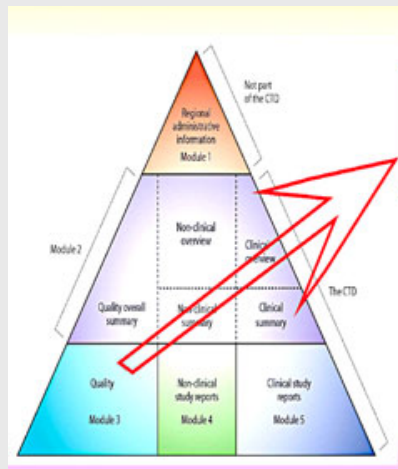

\section{CMC}

\section{Table of con-} ents; Drug sub-

stance; Drug

product

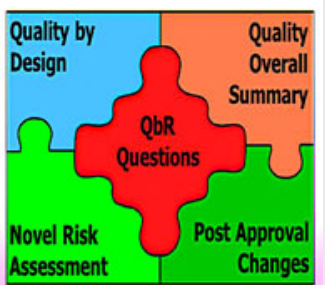

\section{ABOUT AUTHORS}

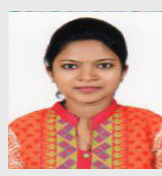

Miss. Akshatha $\mathbf{G}$ is currently pursuing her M Pharm in Pharmaceutical Regulatory Affairs at NGSM Institute of Pharmaceutical Sciences, NITTE (Deemed to be University), Mangalore. Her area of research is comparison and comprehension of drug regulation in both regulated and non-regulated markets.

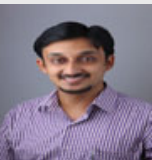

Dr. Anoop Naryanan V is presently working as an Assistant Professor in the Department of Pharmaceutics at NGSM Institute of Pharmaceutical Sciences, NITTE (Deemed to be University), Mangalore. His areas of interest are nanotechnology in drug delivery and regulatory guidelines of various pharmaceutical markets. He owns several papers in reputed research journals into his credit.

Cite this article: Akshatha G, Narayanan AV, Sandeep DS, Charyulu NR. Chemistry Manufacture and Control (CMC) Evaluations of ANDA Submission in the USA. Indian J of Pharmaceutical Education and Research. 2019;53(3):41420. 